\title{
A GLIMPSE AT THE OPERATOR KANTOROVICH INEQUALITY
}

\author{
HAMID REZA MORADI ${ }^{\dagger}$, IBRAHIM HALIL GÜMÜŞ๋ AND ZAHRA HEYDARBEYGI ${ }^{\ddagger}$
}

\begin{abstract}
We show the following result: Let $A$ be a positive operator satisfying $0<m \mathbf{1}_{\mathcal{H}} \leq$ $A \leq M \mathbf{1}_{\mathcal{H}}$ for some scalars $m, M$ with $m<M$ and $\Phi$ be a normalized positive linear map, then

$$
\Phi\left(A^{-1}\right) \leq \Phi\left(m^{\frac{A-M \mathbf{1}_{\mathcal{H}}}{M-m}} M^{\frac{m \mathbf{1}_{\mathcal{H}}-A}{M-m}}\right) \leq \frac{(M+m)^{2}}{4 M m} \Phi(A)^{-1} .
$$
\end{abstract}

Besides, we prove that the second inequality in the above can be squared.

\section{Introduction}

In 1948, L.V. Kantorovich, Soviet mathematician and economist, introduced the well-known Kantorovich inequality [8]. Operator version of Kantorovich inequality was firstly established by A.W. Marshall and I. Olkin, who obtained:

Theorem A. ([10]) Let $A$ be a positive operator satisfying $0<m \mathbf{1}_{\mathcal{H}} \leq A \leq M \mathbf{1}_{\mathcal{H}}$ for some scalars $m, M$ with $m<M$ and $\Phi$ be a normalized positive linear map. Then

$$
\Phi\left(A^{-1}\right) \leq \frac{(M+m)^{2}}{4 M m} \Phi(A)^{-1} .
$$

This note aims to present an improvement of inequality (11). The main result of this note is of this genre:

Theorem 1.1. Let all the assumptions of Theorem A hold. Then

$$
\Phi\left(A^{-1}\right) \leq \Phi\left(m^{\frac{A-M \mathbf{1}_{\mathcal{H}}}{M-m}} M^{\frac{m \mathbf{1}_{\mathcal{H}}-A}{M-m}}\right) \leq \frac{(M+m)^{2}}{4 M m} \Phi(A)^{-1} .
$$

This is proven at the end of Section 2, We start off by fixing some notation: Let $\mathbb{B}(\mathcal{H})$ denote the set of all bounded linear operators on a complex Hilbert space $\mathcal{H}$ with the identity $\mathbf{1}_{\mathcal{H}}$. We extensively use the continuous functional calculus for self-adjoint operators, e.g., see [6, p. 3]. An operator $A$ on $\mathcal{H}$ is said to be positive (in symbol $0 \leq A$ ) if $0 \leq\langle A x, x\rangle$ for all $x \in \mathcal{H}$. We write $0<A$ if $A$ is positive and invertible. For self-adjoint operators $A, B \in \mathbb{B}(\mathcal{H})$,

2010 Mathematics Subject Classification. Primary 47A63, Secondary 46L05, 47A60.

Key words and phrases. Operator inequality, Kantorovich inequality, positive linear maps, log-convex functions. 
we say $A \leq B$ if $0 \leq B-A$. A linear map $\Phi: \mathbb{B}(\mathcal{H}) \rightarrow \mathbb{B}(\mathcal{K})$, where $\mathcal{H}$ and $\mathcal{K}$ are complex Hilbert spaces, is called positive if $\Phi(A) \geq 0$ whenever $A \geq 0$ and is said to be normalized if $\Phi\left(\mathbf{1}_{\mathcal{H}}\right)=\mathbf{1}_{\mathcal{K}}$

A positive function defined on the interval $I$ (or, more generally, on a convex subset of some vector space) is called $\log$-convex if $\log f(x)$ is a convex function of $x$. We observe that such functions satisfy the elementary inequality

$$
f((1-v) a+v b) \leq[f(a)]^{1-v}[f(b)]^{v}, \quad 0 \leq v \leq 1
$$

for any $a, b \in I$. Because of the weighted arithmetic-geometric mean inequality, we also have

$$
f((1-v) a+v b) \leq[f(a)]^{1-v}[f(b)]^{v} \leq(1-v) f(a)+v f(b)
$$

which says that any log-convex function is a convex function.

The following inequality is well known in the literature as the Choi-Davis-Jensen inequality:

Theorem B. $([3,4])$ Let $A \in \mathcal{B}(\mathcal{H})$ be a self-adjoint operator with spectrum $S p(A) \subseteq I$ and $\Phi$ be a normalized positive linear map from $\mathbb{B}(\mathcal{H})$ to $\mathbb{B}(\mathcal{K})$. If $f$ is operator convex function on an interval $I$, then

$$
f(\Phi(A)) \leq \Phi(f(A))
$$

Though in the case of convex function the inequality (4) does not hold in general, we have the following estimate:

Theorem C. ([12, Remark 4.14]) Let $A \in \mathcal{B}(\mathcal{H})$ be a self-adjoint operator with $S p(A) \subseteq[m, M]$ for some scalars $m, M$ with $m<M$ and $\Phi$ be a normalized positive linear map from $\mathbb{B}(\mathcal{H})$ to $\mathbb{B}(\mathcal{K})$. If $f$ is non-negative convex function, then

$$
\frac{1}{\mu(m, M, f)} \Phi(f(A)) \leq f(\Phi(A)) \leq \mu(m, M, f) \Phi(f(A))
$$

where $\mu(m, M, f)$ is defined by

$$
\mu(m, M, f) \equiv \max \left\{\frac{1}{f(t)}\left(\frac{M-t}{M-m} f(m)+\frac{t-m}{M-m} f(M)\right): m \leq t \leq M\right\} .
$$

In Section 2 we prove an analogue of Theorem $\mathrm{C}$ for log-convex functions. The proof of Theorem 1.1 follows quickly from this inequality. In Section [3, inspired by the work of Lin [9], we square the second inequality in (2). 


\section{A refinement of the operator Kantorovich inequality}

An important role in our analysis is played by the following result, which is of independent interest.

Proposition 2.1. Let all the assumptions of Theorem $\mathrm{C}$ hold except the condition convexity which is changed to log-convexity. Then

$$
\Phi(f(A)) \leq \Phi\left([f(m)]^{\frac{M \mathcal{1}_{\mathcal{H}}-A}{M-m}}[f(M)]^{\frac{A-m \mathbf{1}_{\mathcal{H}}}{M-m}}\right) \leq \mu(m, M, f) f(\Phi(A)) .
$$

Proof. It can be verified that if $m \leq t \leq M$, then $0 \leq \frac{M-t}{M-m}, \frac{t-m}{M-m} \leq 1$ and $\frac{M-t}{M-m}+\frac{t-m}{M-m}=1$. Thanks to (3), we have

$$
f(t)=f\left(\frac{M-t}{M-m} m+\frac{t-m}{M-m} M\right) \leq[f(m)]^{\frac{M-t}{M-m}}[f(M)]^{\frac{t-m}{M-m}} \leq L(t),
$$

where

$$
L(t)=\frac{M-t}{M-m} f(m)+\frac{t-m}{M-m} f(M) .
$$

Applying functional calculus for the operator $A$, we infer that

$$
f(A) \leq[f(m)]^{\frac{M 1_{\mathcal{H}}-A}{M-m}}[f(M)]^{\frac{A-m \mathbf{1}_{\mathcal{H}}}{M-m}} \leq L(A) .
$$

Using the hypotheses made about $\Phi$,

$$
\Phi(f(A)) \leq \Phi\left([f(m)]^{\frac{M \mathbf{1}_{\mathcal{H}}-A}{M-m}}[f(M)]^{\frac{A-m \mathbf{1}_{\mathcal{H}}}{M-m}}\right) \leq \Phi(L(A)) .
$$

On account of [12, Corollary 4.12] (the functions $f$ and $g$ there are now $L$ and $f$, respectively), we get

$$
\Phi(f(A)) \leq \Phi\left([f(m)]^{\frac{M \mathcal{1}_{\mathcal{H}}-A}{M-m}}[f(M)]^{\frac{A-m \mathbf{1}_{\mathcal{H}}}{M-m}}\right) \leq \mu(m, M, f) f(\Phi(A)) .
$$

Notice that, although [12, Corollary 4.12] is for matrices, it is also true for operators.

Hence (5) follows.

The following follows immediately from Proposition 2.1. Recall that $f(t)=t^{p},(p<0)$ is log-convex function.

Corollary 2.1. Under the hypotheses of Proposition 2.1, let $p \in(-\infty, 0)$ and $0<m<M$. Then

$$
\Phi\left(A^{p}\right) \leq \Phi\left(m^{p\left(\frac{M \mathbf{1}_{\mathcal{H}}-A}{M-m}\right)} M^{p\left(\frac{A-m \mathbf{1}_{\mathcal{H}}}{M-m}\right)}\right) \leq K(m, M, p) \Phi(A)^{p},
$$

where $K(m, M, p)$ is the generalized Kantorovich constant defined by

$$
K(m, M, p) \equiv \frac{m M^{p}-M m^{p}}{(p-1)(M-m)}\left(\frac{p-1}{p} \frac{M^{p}-m^{p}}{m M^{p}-M m^{p}}\right)^{p} .
$$


Remark 2.1. We would like to mention that (5) can be regarded as an improvement of [7, Theorem 1.5] (see also [11, Lemma 2]).

After the previous technical intermission, we return to the main subject of this section, the proof of the inequality (2).

Proof of Theorem 1.1. This follows from Corollary 2.1 by putting $p=-1$. We should point out that $K(m, M,-1)=\frac{(M+m)^{2}}{4 M m}$.

Can the second inequality in (2) be squared? Responding to this question is the main motivation of the next section.

\section{Squaring refinement of the operator Kantorovich inequality}

We will need the following lemmas.

\section{Lemma 3.1.}

(i) [2, Theorem 1] Let $A, B>0$. Then the following norm inequality holds:

$$
\|A B\| \leq \frac{1}{4}\|A+B\|^{2} .
$$

(ii) [1, Theorem 3] Let $A, B \geq 0$ and $1 \leq r \leq \infty$. Then

$$
\left\|A^{r}+B^{r}\right\| \leq\left\|(A+B)^{r}\right\|
$$

Lemma 3.2. For each $m \leq t \leq M$, we have

$$
t+m M m^{\frac{t-M}{M-m}} M^{\frac{m-t}{M-m}} \leq M+m .
$$

Proof. Because of the weighted arithmetic-geometric mean inequality

$$
\begin{aligned}
t+m M m^{\frac{t-M}{M-m}} M^{\frac{m-t}{M-m}} & =t+m^{\frac{t-m}{M-m}} M^{\frac{M-t}{M-m}} \\
& \leq t+\frac{t-m}{M-m} m+\frac{M-t}{M-m} M \\
& =M+m,
\end{aligned}
$$

which finishes the proof.

Now we are at the position to state our main result.

Theorem 3.1. Let all the assumptions of Theorem A hold. Then

$$
\Phi\left(m^{\frac{A-M \mathbf{1}_{\mathcal{H}}}{M-m}} M^{\frac{m \mathbf{1}_{\mathcal{H}}-A}{M-m}}\right)^{p} \leq\left(\frac{(M+m)^{2}}{4^{\frac{2}{p}} M m}\right)^{p} \Phi(A)^{-p} \quad \text { for } 2 \leq p<\infty .
$$


In particular

$$
\Phi\left(m^{\frac{A-M \mathbf{1}_{\mathcal{H}}}{M-m}} M^{\frac{m \mathbf{1}_{\mathcal{H}}-A}{M-m}}\right)^{2} \leq\left(\frac{(M+m)^{2}}{4 M m}\right)^{2} \Phi(A)^{-2} .
$$

Proof. The idea of the proof is similar to [5, Theorem 3]. It is easy to see that if $A, B>0$ and $\alpha>0$, then

$$
A \leq \alpha B \quad \Leftrightarrow \quad\left\|A^{\frac{1}{2}} B^{-\frac{1}{2}}\right\| \leq \alpha^{\frac{1}{2}}
$$

So we are done if we can show

$$
\left\|\Phi\left(m^{\frac{A-M \mathbf{1}_{\mathcal{H}}}{M-m}} M^{\frac{m \mathbf{1}_{\mathcal{H}}-A}{M-m}}\right)^{\frac{p}{2}} \Phi(A)^{\frac{p}{2}}\right\| \leq \frac{(M+m)^{p}}{4 M^{\frac{p}{2}} m^{\frac{p}{2}}} .
$$

On account of Lemma 3.2, it follows that

$$
\Phi(A)+m M \Phi\left(m^{\frac{A-M \mathbf{1}_{\mathcal{H}}}{M-m}} M^{\frac{m \mathbf{1}_{\mathcal{H}}-A}{M-m}}\right) \leq(M+m) \mathbf{1}_{\mathcal{H}}
$$

By direct calculation,

$$
\begin{aligned}
& \left\|m^{\frac{p}{2}} M^{\frac{p}{2}} \Phi\left(m^{\frac{A-M \mathbf{1}_{\mathcal{H}}}{M-m}} M^{\frac{m \mathbf{1}_{\mathcal{H}}-A}{M-m}}\right)^{\frac{p}{2}} \Phi(A)^{\frac{p}{2}}\right\| \\
& \leq \frac{1}{4}\left\|m^{\frac{p}{2}} M^{\frac{p}{2}} \Phi\left(m^{\frac{A-M \mathbf{1}_{\mathcal{H}}}{M-m}} M^{\frac{m \mathbf{1}_{\mathcal{H}}-A}{M-m}}\right)^{\frac{p}{2}}+\Phi(A)^{\frac{p}{2}}\right\|^{2} \quad \text { (by Lemma 3.1 (i)) } \\
& \leq \frac{1}{4}\left\|\left(m M \Phi\left(m^{\frac{A-M \mathbf{1}_{\mathcal{H}}}{M-m}} M^{\frac{m \mathbf{1}_{\mathcal{H}}-A}{M-m}}\right)+\Phi(A)\right)^{\frac{p}{2}}\right\|^{2} \quad \text { (by Lemma 3.1 (ii)) } \\
& \quad=\frac{1}{4}\left\|m M \Phi\left(m^{\frac{A-M \mathbf{1}_{\mathcal{H}}}{M-m}} M^{\frac{m \mathbf{1}_{\mathcal{H}}-A}{M-m}}\right)+\Phi(A)\right\|^{p} \\
& \leq \frac{(M+m)^{p}}{4} \quad(\text { by (10) }) .
\end{aligned}
$$

This proves (9).

Acknowledgements. We would like to thank the referee(s) for carefully reading our manuscript and for giving such constructive comments which substantially helped to improve the quality of the paper.

\section{REFERENCES}

[1] T. Ando, X. Zhan, Norm inequalities related to operator monotone functions, Math. Ann. 315 (1999), $771-780$.

[2] R. Bhatia, F. Kittaneh, Notes on matrix arithmeticgeometric mean inequalities, Linear Algebra Appl. 308 (2000), 203-211.

[3] M.D. Choi, A Schwarz inequality for positive linear maps on $C^{*}$-algebras, Illinois J. Math. 18 (1974), 565574.

[4] C. Davis, A Schwarz inequality for convex operator functions, Proc. Amer. Math. Soc. 8 (1957), $42-44$. 
[5] X. Fu, C. He, Some operator inequalities for positive linear maps, Linear Multilinear Algebra. 63(3) (2015), $571-577$.

[6] T. Furuta, J. Mićić-Hot, J. Pečarić, Y. Seo, Mond-Pečarić method in operator inequalities, Element, Zagreb, 2005.

[7] T. Furuta, Operator inequalities associated with Hölder-McCarthy and Kantorovich inequalities, J. Inequal. Appl. 1998.2 (1998): 234521.

[8] L.V. Kantorović, Functional analysis and applied mathematics, Uspekhi Math. Nauk. 3 (1948), Translated from the Russian by Curtis D. Benster, Nat. Bur. Stand ards, Report No. 1509., March 7, 1952.

[9] M. Lin, On an operator Kantorovich inequality for positive linear maps, J. Math. Anal. Appl. 402 (2013), $127-132$.

[10] A.W. Marshall, I. Olkin, Matrix versions of Cauchy and Kantorovich inequalities, Aequationes Math. 40 (1990), 89-93.

[11] J. Mićić, J. Pečarić, Order among power means of positive operators, II, Sci. Math. Japon. 71(1) (2010), 93-109.

[12] J. Mićić, J. Pečarić, Y. Seo, M. Tominaga, Inequalities for positive linear maps on Hermitian matrices, Math. Inequal. Appl. 3 (2000), 559-591.

\footnotetext{
${ }^{\dagger}$ Young Researchers and Elite Club, Mashhad Branch, Islamic Azad University, Mashhad, Iran.

E-mail address: hrmoradi@mshdiau.ac.ir

${ }^{\ddagger}$ Department of Mathematics, Faculty of Arts and Sciences, Adiyaman University, Adiyaman, Turkey.

E-mail address: igumus@adiyaman.edu.tr

$\S$ Department of Mathematics, Mashhad Branch, Islamic Azad University, Mashhad, Iran.

E-mail address: zheydarbeygi@yahoo.com
} 